\title{
Retinal Blood Vessels and Optic Disc Segmentation Using U-Net
}

\author{
S. Alex David $\mathbb{D}^{1}{ }^{1}$ C. Mahesh, ${ }^{1}$ V. Dhilip Kumar, ${ }^{1}$ Kemal Polat $\mathbb{D}{ }^{2}$ Adi Alhudhaif $\mathbb{D}{ }^{3}$ \\ and Majid Nour ${ }^{4}$ \\ ${ }^{1}$ Vel Tech Rangarajan Dr Sagunathala $R$ \& D Institute of Science and Technology, Chennai, India \\ ${ }^{2}$ Bolu Abant Izzet Baysal University, Faculty of Engineering, Department of Electrical and Electronics Engineering, Bolu, Turkey \\ ${ }^{3}$ College of Computer Engineering and Sciences in Al-kharj, Prince Sattam Bin Abdulaziz University, Al-Kharj, Saudi Arabia \\ ${ }^{4}$ Department of Electrical and Computer Engineering, Faculty of Engineering, King Abdulaziz University, Jeddah 21589, \\ Saudi Arabia
}

Correspondence should be addressed to Kemal Polat; kpolat@ibu.edu.tr

Received 4 January 2022; Accepted 18 January 2022; Published 14 February 2022

Academic Editor: Nagarajan DEIVANAYAGAMPILLAI

Copyright (C) 2022 S. Alex David et al. This is an open access article distributed under the Creative Commons Attribution License, which permits unrestricted use, distribution, and reproduction in any medium, provided the original work is properly cited.

\begin{abstract}
A color fundus image is a photograph obtained using a fundus camera of the inner wall of the eyeball. In the image, doctors may see changes in the retinal vessels, which can be used to diagnose various dangerous disorders such as arteriosclerosis, some macular degeneration related to age, and glaucoma. To diagnose certain disorders as early as possible, automatic segmentation of retinal arteries is used to help the doctors. Also, it is a challenge for the medical community to analyze the image with the right procedure to diagnose the disorders with high accuracy. Furthermore, this will help the doctor to make the right decision on effective treatment. Hence, the authors have implemented an enhanced architecture called U-Net to segment retinal vessels in this paper. The proposed conventional U-Net permits using all the accessible spatial setting information by adding the multiscale input layer and a thick square to the conventional U-Net in terms of improving the accuracy level of image segmentation. It achieved 95.6\% accuracy with a comparatively traditional U-Net model. Moreover, the segmentation results have proved that the proposed approach outperformed in detecting most complex low-contrast blood vessels even when they are very thin. The task of segmenting vessels in retinal images is known as retinal vessel segmentation. Blood vessel density can be assessed using dense pixel values. Data augmentation and analytics play a major role in building the true value of eye blood vessels for medical diagnosis. The proposed method is very promising in the automatic segmentation of retinal arteries.
\end{abstract}

\section{Introduction}

A special fundus camera is used to photograph the color fundus image, which is the inside and back surface of the eyeball. The picture is obtained in a painless and noninvasive manner. In fundus images, retinal vascular alterations that can be used to diagnose various significant disorders such as macular degeneration, arteriosclerosis, and glaucoma [1] can be identified. Manually analyzing blood vessels in fundus photographs, on the contrary, is time-consuming and laborintensive for clinicians $[2,3]$. They can reduce time and effort by automating the segmentation of retinal layers.

Some of the retinal nerve fibers present in the oculus dexter (OD) transfer the images taken from the eyeball first converted into visual signals and transferred immediately to the head of the body called the brain. The cells in the retina called photoreceptors are found in the OD of the retina, which aid vision. The "blind spot," as the OD dubbed it, was created as a result of this. The brightest area of the retina is oculentum (OC) [4], which is located at the innermost part of the OD [5]. In healthy people, an OC covers about $30 \%$ of the disc.

To identify the effect of glaucoma in the eye, different models of eye images are captured such as the fundus image by using various methods such as magnetic resonance imaging (MRI) and optical coherence tomography (OCT); however, in glaucoma detection, fundus pictures are one of the most suggested methodologies. These are the eye images taken using a camera known as a fundus camera. Other elements such as the veins, optic cup and disc, fovea, macula, artery, and other retinas will be easily portrayed in fundus images [6]. It has also been demonstrated that using the fundus images, ocular illnesses can be anticipated quickly. 
In fundus imaging, the brightest portion is the neuroretinal rim and OC present in the OD. One of the inner parts of the OD is OC, where OC's outer limit is the neuroretinal rim. Optic cupping occurs as the size of the OC grows larger. Glaucoma can be easily recognized by locating the optic nerve cupping. Many metrics will be used to diagnose glaucoma, but the value of cup-disc ratio (CDR) is the best diagnostic, calculated by measuring the distance between the $\mathrm{OD}$ and OC. At some time, there is a chance of OC size which may be higher than the actual size; at this place, glaucoma is diagnosed by experts [7]. We need to segment both OD and OC to calculate the CDR value, but conventional manual procedures take longer to segment and produce inaccurate results. From the result, OC and OD autosegmentation is not accurate also; it is a critical process in the detection of glaucoma [8]. Figure 1 depicts both normal healthy and glaucoma-affected eyes with the OD and OC highlighted in dotted lines.

Figure 1(a) depicts a healthy eye, and Figure 1(b) depicts a suspect eye for glaucoma. The optic disc is represented by the outside dotted lines, while the optic cup is represented by the inner dotted line.

1.1. Motivation. Glaucoma is a most referred disease in the ophthalmology division of the medical sector. This disease will be more dangerous when it is untreated, and it leads to permanent blindness. At this situation, the unwanted fluid at the place of the anterior portion of the retina will make a high pressure in the eye and will become a cause of optic nerve damage [9]. The survey indicates that the total number of cases related to glaucoma will rise to nearly $80 \%$ worldwide by 2022 . Also, the studies depict that people from the age of 60 will have high risk on this disease. Hence, it is necessary to detect and diagnosis this disease at the earliest to avoid the worst circumstances.

The segmentation of retinal vessels has obtained a lot of attention, but there are still a lot of challenges. For starters, blood vessels come in a variety of forms, diameters, and grey degrees. Second, the dissimilarity between some vessels and their surrounding is relatively low [10]. At last, there are some changes which manifest themselves with bright patches among dark and thin spaces, resembling blood vessels in appearance.

The context fundus has arrived from the Latin word, and it means that a portion of the eyeball is positioned opposite the eye pupil [11-14]. Hence, an image taken by using a high-density camera on the interior part of the eye will be referred for identifying the fundus. A popular technique, namely, funduscopy, is used to capture the fundus image. However, the medical industry has developed many approaches such as laser scanning polarimetry and optical coherence tomography in recent years, but the cost of the medical equipment is very high, and many hospitals and medical centers may not be affordable. The reachability of these facilities in rural areas would be challenging for them [15-20].

By considering these challenges, this paper aims to propose alternative and cost-effective methods for the detection of glaucoma using fundus images with the help of an optic disc. An optic disc (OC) is a bright part at the center of the retina of the eye, and it is resided in the optic disk. In the situation of glaucoma case, there will be a change in the structure of the optic cup. This situation is commonly known as cupping. Hence, the ratio called cupto-disc ratio (CDR) which will be arrived from the structural changes of the optic cup is used as meaning information and used as an indicator to identify and diagnose the glaucoma condition.

\subsection{Major Contribution of the Optimized Pretrained Model.} There are supervised and unsupervised segmentation algorithms available (elaborated in Section 2). Neural network-based algorithms are supervised methods that can considerably enhance vessel segmentation accuracy [21]. On the contrary, segmentation of thin vessels is harder because their nearby pixels are very low in quality. The proposed enhanced U-Net model for segmenting retinal vessels produces good accuracy and superior segmentation results for thin vessels in retinal images. The following are the primary contributions of this work:

(1) Different scale picture patches are supplied into the network as inputs, allowing it to learn more multiscale data. More spatial context data are obtained in the U-Net model using dense blocks. As a result of these tactics, the proposed method can detect more vessel pixels, allowing for better segmentation of thin vessels.

(2) When compared to the standard or "vanilla" U-Net design, the proposed method improves the sensitivity and accuracy of vessel segmentation. When it comes to segmenting narrow vessels, the proposed method offers evident advantages in both qualitative and quantitative assessments.

(3) By calculating different measuring attributes such as sensitivity and specificity, the suggested model achieves excellent accuracy. The sensitivity of the geometric mean was also assessed in order to arrive at the closest result. In comparison to previous retinal vascular segmentation methods, the output is quite good. Our method outperforms the state-ofthe-art method in terms of the receiver operating characteristic curve (OCR).

This paper is coordinated as follows: Section 2 talks about some refined vascular division procedures. Section 3 dives into the subtleties of the proposed procedure, while Section 4 examines the datasets utilized in the investigations just as the trial boundaries. The outcomes from tests are examined in Section 5. Finally, Section 6 ends up with a conclusion.

\section{Related Work}

The segmentation of retinal vessels has received a lot of attention. Unsupervised techniques and supervised methods are the two types of methods. 


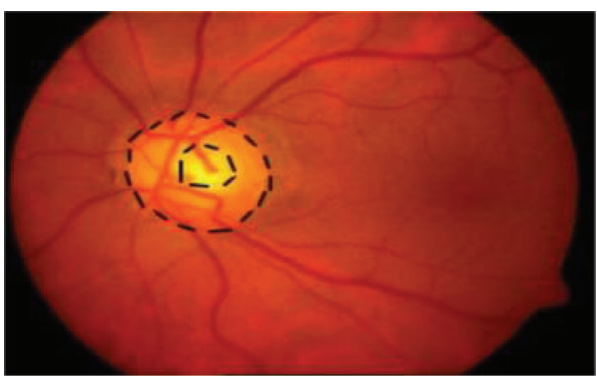

(a)

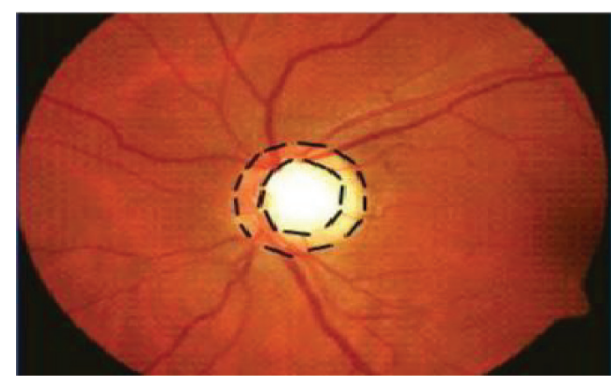

(b)

Figure 1: (a) Healthy eye image. (b) Glaucomatous eye image.

Prior information of the vascular structure or grey characteristics is used in the unsupervised algorithms. However, due to their diverse presentations, it is difficult to include all vascular structures. Model-based approaches, matching filters, and morphological methods are among the unsupervised methods. Adaptive thresholding was used [22] to identify pixels of vessels by the iterative process. From the retinal picture, regional data are extracted by using the contour model [22]. To segment retinal vessels, combine both region growth algorithms with the level set [23]. A new 3 -D-oriented filter method is proposed to enhance the translation of color to grey with basics of principle component analysis [24, 25]. A double-sided thresholding approach was newly created, and a Gabor filter was employed to enhance photos. The Hessian matrix and binary pictures are combined together to make an entropy-maximizing threshold for improved vessels. Guo et al. and Khan et al. $[26,27]$ used a multiscale line detector to segment retinal arteries. A matching filter with signed integers is used to improve the difference among both vascular and nonvascular pixels. A number of contrast-sensitive approaches for improving the sensitivity of existing retinal vascular segmentation algorithms are implemented [28, 29].

Recent research has primarily focused on deep featurebased approaches. Without any prior experience, they can learn hierarchical features automatically. Postprocessing is usually not required for segmentation results. These papers suggest several neural network topologies.

The following are some of the earlier studies on the prediction of various eye diseases by using different techniques.

The authors in [30-32] looked on difficulties in describing the location of the retinal structure in terms of image. The image pixel distance has been forecasted based on a specific location from the set of picture feature using the $\mathrm{KNN}$ model. An ideal $K$-value is not determined perfectly because of the regression model [33-36]. Also, to improve the recognition performance, the type of metric used is unfair with the pixel-based distance method.

A well fined and tuned optic disc (OD) and its locators are grouped into a complicated system. Both quality of prediction and performance ratio are improved in terms of accuracy rate. Later on, it was assumed to be a self-contained object-location difficulty. A single candidate for OD focal point fixation isolated by soe measurement and classified as self-contained dominant element using by a higher precision value.
Some of the measurements isolate a single candidate for OD focal point fixation, which is limited by a higher precision value and is classified as the voting standard's selfcontained dominant element [37-40]. They employed likelihood maps in the recognition procedure. However, throughout the test period, the difficulty with this strategy is that it is both highly one-sided and computationally expensive.

In other circumstances, the visual judgment is based on some basic high priority knowledge and restricted potential when it comes to detecting tiny distinctions and characterizing novel image makers [41-44]. Some of the novel data analytic and imaging techniques are used to improve the quantitative information and also reduce the reader variations by applying any of the following [45, 46]: machine learning (ML), deep learning (DL), and radionics. It also improves prognostic and diagnostic accuracy ratios while minimizing preconception.

Glaucoma and retinal fundus screening and diagnosis system is fully automated [1]. A three-step procedure is followed to achieve the above system. Initially, similar template and condition techniques are combined together to detect the OD. Second, texture-based segmentation is used with the model to extract both retinal OD and OC. At last, third, they used the CDR to distinguish between healthy and glaucomatous individuals. The expert's trained simulation results show $98 \%$ accuracy on classifying the glaucomatous or designated healthy eye [47]. The demonstrated results of the proposed model have higher accuracy than those of the existing model. All they intend to use is a cutting-edge system to investigate the use of deep learning to improve the performance of early glaucoma screening and diagnosis.

In addition, numerous computational techniques in artificial intelligence are used to diagnose diseased locations. A deep learning method is used with some other fully automated techniques [48]. The array-based technique is used in conjunction by combining both VGG representation and fully connected layers. An improved technique suggested for detecting impacted regions using medical imaging is proposed [49], transformation is performed to denoise the image, and an optimizing approach is utilized to take out needed data from the image. These characteristics finally come up from an image from its texture, size, and shape. Following feature extraction, the priority technique picks $70 \%$ of the features. Finally, an SVM classifier is used to classify the image $[50,51]$. 
2.1. Segmentation of the Optic Disc. In the retina, a circular region which is in dark yellow is the optic disk. The OD has a slightly circular form, although it is unique to everyone [52]. The head of the optic nerve examines a huge number of neurons. Since it lacks in color-detecting photoreceptors like rods and cones, it is called as "blind spot." For segmenting the optic disk, various methods were discussed.

In order to segment the optical disc, a new templatebased approach is used [53]. The modified variance picture was used to pinpoint the disc and retina rim part. The optic nerve head is outlined in shape utilizing the watershed technique and is able to obtain $90 \%$ accuracy by employing this strategy [54]. A reliable mechanism for optical disc location is also used in this mechanism. The following four processes can be used for optic disc circular boundary measurement: OD localization, filtering by the nonlinear method, Canny edge detector for edge extraction, and circular boundary identification using the Hough transform [55-57]. This results in the OD localization accuracy of $97 \%$ and OD boundary extraction accuracy of $82 \%$. 99.1\% accuracy is reached by using principle component analysis (PCA) for optical disc localization; also, border identification obtained an accuracy of $94 \%$ using the active shape model (ASM).

A template matching technique is applied to identify the figure of a picture using an active contour model. For classifying images, a Bayesian classifier is used, which effectively categorizes images based on pixel-based classification [58].

Conventional autoencoder (CAE) is used to extract unlabeled picture features in this case. The autoencoder (AE) assists in the development of a pretrained model by learning features from the unlabeled input. After that, transfer learning was used to train the constructed model, which resulted in a 96\% dice metric accuracy [59-61]. However, training both models took longer. Image segmentation is a challenging task in medical imaging. Therefore, deep learning is being used extensively in picture segmentation. A full network channel for segmentation can be implemented for obtaining good results [29]. The implemented model aids in the retention of a greater number of picture features as well as the restoration of the image for a longer period of time. As a result, this approach aids in the correct segmentation of the optic disc. However, when it comes to segmenting the optic cup, the same procedure fails.

\section{Methodology}

3.1. Overview of the Methodology. The color retinal images are first preprocessed to create enhanced grey images. The image patches around the vessel pixels are then retrieved and reutilized for $\mathrm{U}-\mathrm{Net}$ architecture improvement. The picture prospect map is created by combining the patch probability maps generated by the enhanced U-Net. Binary segmentation is used to produce the result of the last segmentation process. Figure 2 shows the workflow of the proposed method which identifies the segmentation results from the color retinal image.
The network architecture is patch-based, as depicted in Figure 3. The encoder path is on the left, while the decoder path is on the right, as in the original U-Net architecture. In all encoder layers, the convolution process is conducted with a rectified linear unit (ReLU) and batch normalization to build multichannel encoder feature maps. After that, the downsampling procedure is performed. Next, the feature maps are upsampled using the deconvolution layer in the decoder pipeline. By using skip connections, the feature map of the encoder path is interconnected with the appropriate feature map's upsample decoder [32, 62]. Finally, a softmax function is used to activate the feature maps generated by the final decoder layer. One channel probability is obtained for vessels, while the other is determined for nonvessels.

The existing architecture for segmentation can be improved on the basis of the following two aspects:

(1) Input layer among many scales: the use of multiscale input has been shown to improve segmentation quality. For downsampling, the image average pooling layer is used and obtains the output as pictures with different scales which are fed into each layer's encoder path. Dissimilar to past endeavors, which put multiscale pictures in multistream networks and intertwined the result guide of each organization as the last result, this work utilizes a solitary result map for all organizations [63].

(2) Block with a lot of density: we employ a three-layer dense block, as illustrated in Figure 4. For selecting inputs of every layer, the earlier layer's feature maps are used; also, for subsequent layers, their own feature maps are used. First two layers perform batch normalization, ReLU activation, and convolution as part of a transformation. Only ReLU activation is implemented in the last layer [64-68].

3.2. Loss Function for the Optimizer. For optimizing the network, Adam's law is used. This law needs gradients of the first order with minimal memory. To determine the individual adaptive learning rates of different parameters, the estimations of 1 st and 2 nd instants of slopes were used.

The discrepancy between the expected and true distributions of the picture pixel is measured using binary crossentropy. The following is the definition of the loss function:

$$
\text { loss }=-\frac{1}{N} \sum_{i=1}^{N}[M i \log (Q i)+(1-M i) \log (1-Q i)] \text {. }
$$

Here, $N$ indicates no samples taken, $M i$ defines the accurate label at the $i$ th sample, and the expected probability of the sample at the $i$ th position is defined by $Q i$.

\section{Experimental Setup}

4.1. Data Taken for the Experiment. The suggested U-Net architecture approach is tested with the DRIVE [69] dataset, which is freely available. DRIVE is made up of 40 retinal images, each of which was taken at 768584 pixels, with eight 


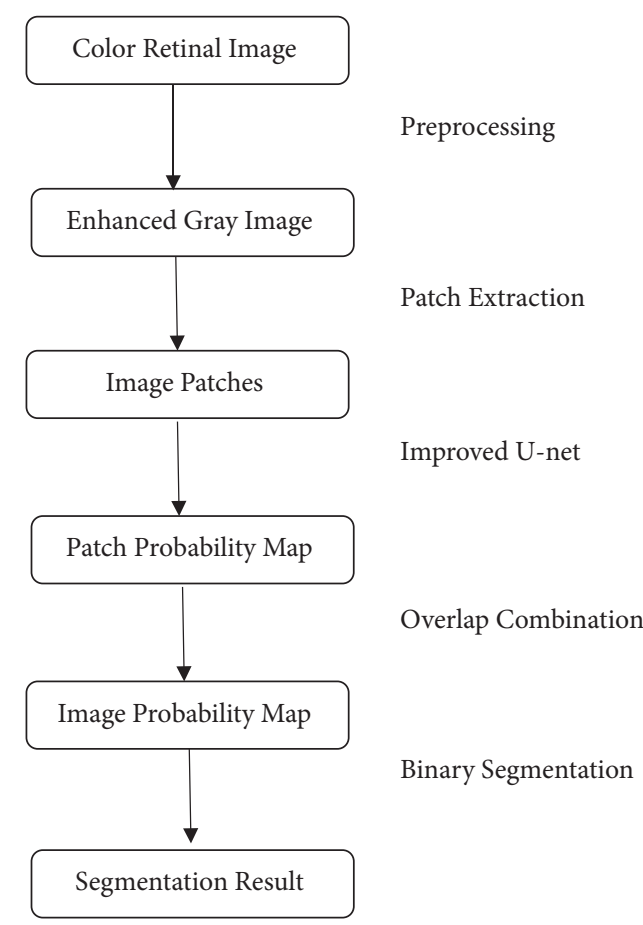

Figure 2: Proposed method's workflow.

bits for each color plane taken. Thirty-three photos show no symptoms of diabetic retinopathy, whereas seven exhibit mild signs of premature diabetic retinopathy. In the experiment, datasets are separated among 2 clusters. Among 40 images, 20-20 are taken for training and testing, respectively. As ground truth, each training image contains one manual segmented image, where two images are manually segmented in each test data. Most prior techniques employ the first one as the ground truth for evaluation $[25,42]$. We use the same approach as other methods to ensure a fair comparison.

4.2. Parameter Setting. The deep learning implementation API Keras id is used to execute the proposed method, and the Adam optimizer uses 0.0001 learning rate for network optimization. All the training images are doubled at the whole training level [70]. In addition, 40 retinal color pictures are improved and turned to grayscale. From the image, 96-bit patches are extracted as 20,484 and used as network inputs.

In network training, the cluster size is set as 25 and executed as three epochs. The images are converted as grayscale images and follow the remaining as like the training phase. From the grey image, 9696 patches were extracted using a stride with 5.1 in both sides [71]. Next, fixed forecast maps are acquired and covered to frame an expectation picture. At last, with a limit of 0.5 , binary segmentation has been implemented.

4.3. Evaluation Metrics. Segmentation results are registered by a picture veil characterized by the field perspective on the pictures. Precision can be estimated by every one of the measurements such as sensitivity (SE), specificity (SP), and accuracy (ACC) and mean of affectability and explicitness. We employ to assess the strategy ( $G$-mean). The following is a list of their definitions:

$$
\begin{aligned}
\text { sensitivity }(\mathrm{SE}) & =\frac{\operatorname{true} \text { positive }(\mathrm{TP})}{\text { true positive }(\mathrm{TP})+\text { false negative }(\mathrm{FP})}, \\
\text { specificity }(\mathrm{SP}) & =\frac{\operatorname{true~negative~}(\mathrm{TN})}{\text { true negative }(\mathrm{TN})+\text { false positive }(\mathrm{FP})}, \\
\text { accuracy } & =\frac{\operatorname{true~positive~}(\mathrm{TP})+\text { true negative }(\mathrm{TN})}{\text { true positive }(\mathrm{TP})+\text { false negative }(\mathrm{FN})+\text { true negative }(\mathrm{TN})+\text { false positive }(\mathrm{FN})}, \\
G-\text { mean } & =\sqrt{\text { sensitivity }(\mathrm{SE})+\text { specificity }(\mathrm{SP})}
\end{aligned}
$$

True positives (TPs) characterize vessels that are perceived accurately as vessels, and true negatives are nonvessel pixels recognized as nonvessels, whereas the false positives are pixels of nonvessels which are recognized as vessels, and the false negatives are pixels wrongly delegated nonvessels.

Sensitivity (SE) is utilized here to ascertain how accurately pixels are characterized and mentioned in (2). Meanwhile, in (3), the number of accurately characterized nonvessel pixels is represented by specificity (SP). All around, the accurately ordered picture pixels are characterized in accuracy by using (4), which determines the performance of the classifier model [72]. A geometric mean is calculated between the sensitivity and specificity which lies between 0 and 1 . Furthermore, the suggested method's performance is assessed using a ROC curve; also, the area under the ROC (ARC) is calculated to define implementation of the classifier. When a 


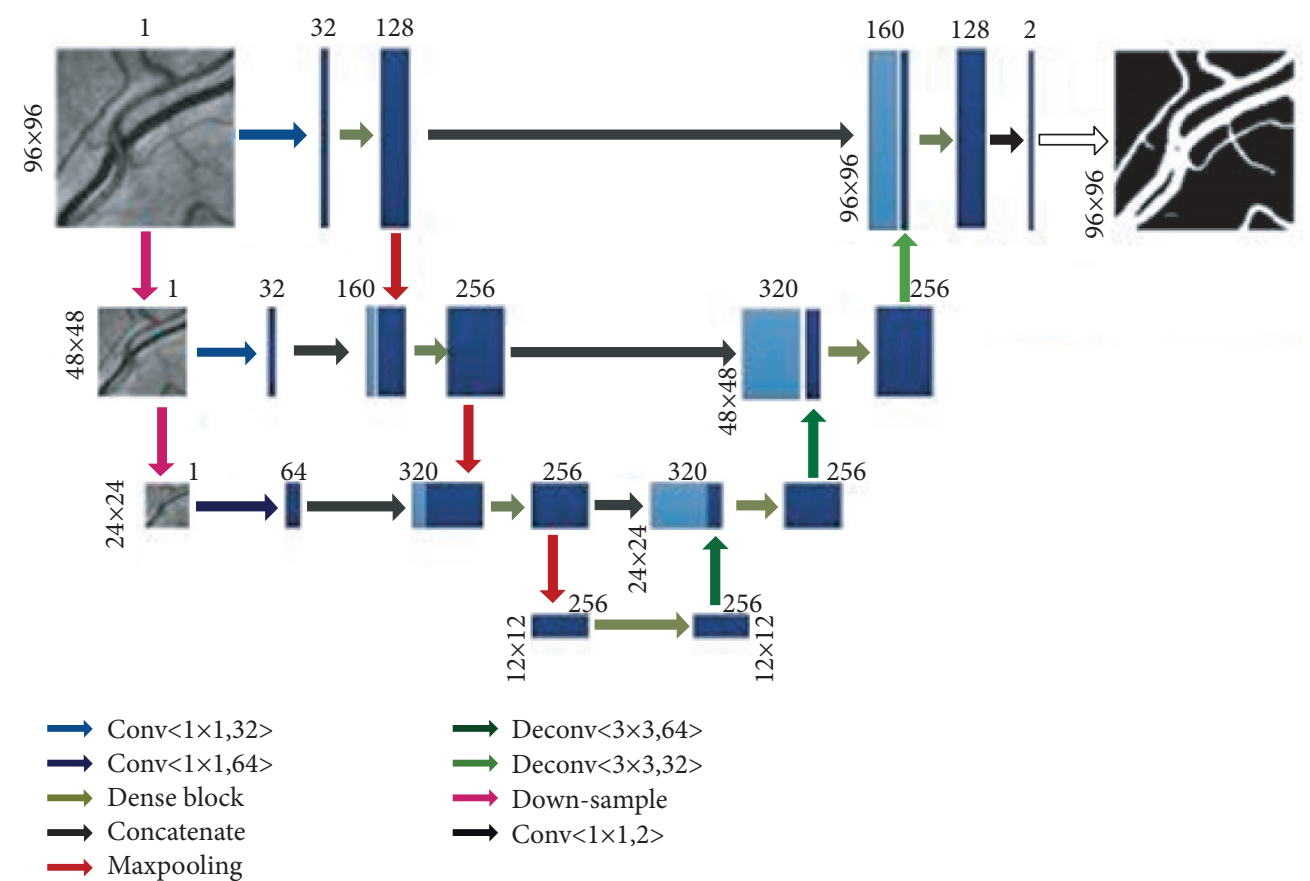

Figure 3: Improved U-Net architecture.

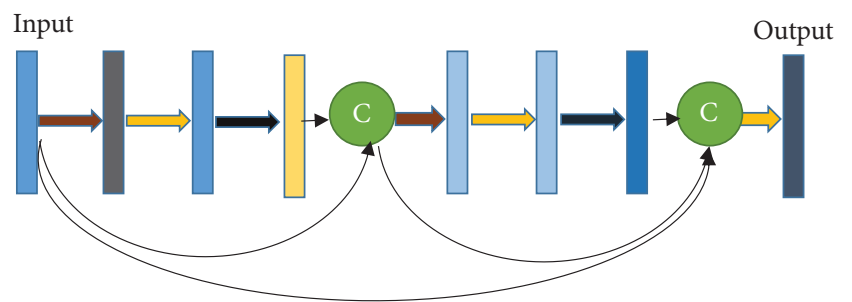

$\begin{array}{ll}\longrightarrow \text { Batch normalization } & \longrightarrow \text { Convolution } \\ \longrightarrow \text { ReLU } & \text { (C) Concatenate }\end{array}$

Figure 4: Architecture of dense blocks.

high AUC value performance of the classifier is identified, if it is 1 , then the classifier is faultless.

\section{Results and Discussion}

5.1. Performance of U-Net. Table 1 shows the strategy's presentation on the DRIVE dataset, with the second human spectator results filling in as a benchmark. Our strategy's normal sensitivity (SE), specificity (SP), accuracy (ACC), and geometric mean ( $G$-mean) by using equation (5), individually, are $0.82,0.98,0.9661$, and 0.8992 . The four estimations are generally better than the subsequent spectators.

Segmentation of two images is defined in Figure 5. Figures 5(a) and 5(e) are the original retinal images. After applying mask, resulting images are shown in Figures 5(b) and 5(f). Human observer result images are shown in Figures 5(c) and 5(g). Finally, the proposed U-Net architecture result is shown in Figures 5(d) and 5(h). From Figure 5, one can conclude that the proposed method provides higher accuracy in classification than others.
5.2. Performance Comparison of the Proposed Method. To present the proposed architecture performance, results are compared from other researchers' work from the net source with the proposed method and plotted in Table 2. As a result, the values of all sensitivity (SE), specificity (SP), accuracy (ACC), and geometric mean ( $G$-mean) are improved in the proposed method U-Net model.

The result of SE and ACC of each image segmentation is shown in Figures 6(a) and 6(b). In Figure 6(a), the proposed model's sensitivity is higher than the existing model. The accuracy of the proposed model is shown in Figure 6(b) which is also comparatively higher than other methods.

In Figure 7, the curve of ROC is exhibited, and the AUC values are displayed. The improved U-Net's ROC curve is nearer to the high left corner than the existing U-Net, as seen in Figure 6. The upgraded U-Net has an AUC of 0.9796, which is higher than the existing method (0.9553). This shows that the proposed method strategy produces a more accurate classifier.

To perform the accuracy comparison, the input image is split into two sections in the case of thin vessel segmentation. Thin vessels are grouped together which have diameter less than five pixels. Figure 8(a) shows the initial test ground truth image, Figure 8 (b) shows the ground truth for thin vessels, and Figure $8(\mathrm{c})$ shows the ground truth for large vessels. Thin vessels' ground truth map is calculated to find the sensitivity of both existing and proposed improved U-Net which is shown in Figure 9. An improved U-Net is used to detect the thin vessels, as shown in Figure 10. The proposed U-Net is also used to segment the thin vessels; even their contrast is very poor.

5.3. Computational Time. The model is run by an Intel Xeon E5-2683 2.0 GHz processor and a NVIDIA Titan XP GPU framework. A retinal picture takes about 21.5 seconds to 
TABLE 1: Performance comparison for retinal blood vessel segmentation.

\begin{tabular}{lcccc}
\hline Model & Sensitivity $(\mathrm{SE})$ & Specificity $(\mathrm{SP})$ & Accuracy $(\mathrm{ACC})$ & Geometric mean $(G-\mathrm{mean})$ \\
\hline Reference human observer & 0.77 & 0.97 & 0.9010 & 0.8141 \\
Proposed U-Net method & 0.82 & 0.98 & 0.9661 & 0.8992 \\
\hline
\end{tabular}

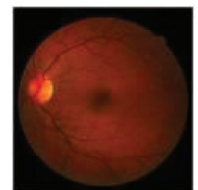

(a)

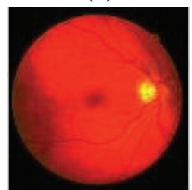

(e)

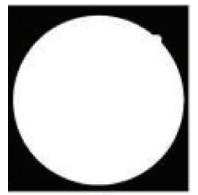

(b)

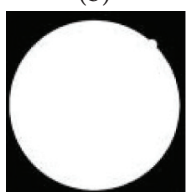

(f)

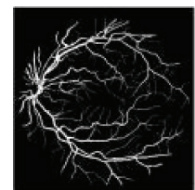

(c)

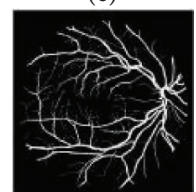

(g)

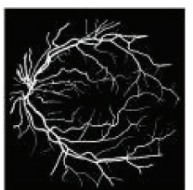

(d)

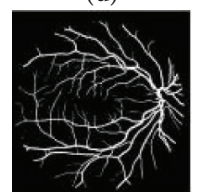

(h)

FiguRE 5: Retinal images' segmentation: (a, e) retinal images in color; (b, f) result from masking; (c, g) human reference; (d, h) result of the proposed U-Net architecture.

TABLE 2: Comparison between the traditional and proposed U-Net.

\begin{tabular}{lcccc}
\hline Model & Sensitivity & Specificity & Accuracy & Geometric mean \\
\hline Vanilla U-Net & 0.712 & 0.992 & 0.9557 & 0.8752 \\
Proposed U-Net & 0.82 & 0.98 & 0.9661 & 0.8992 \\
\hline
\end{tabular}

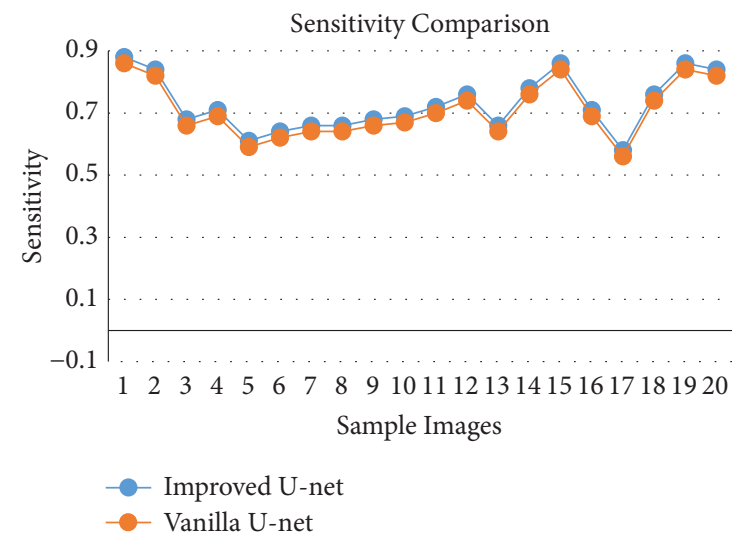

(a)

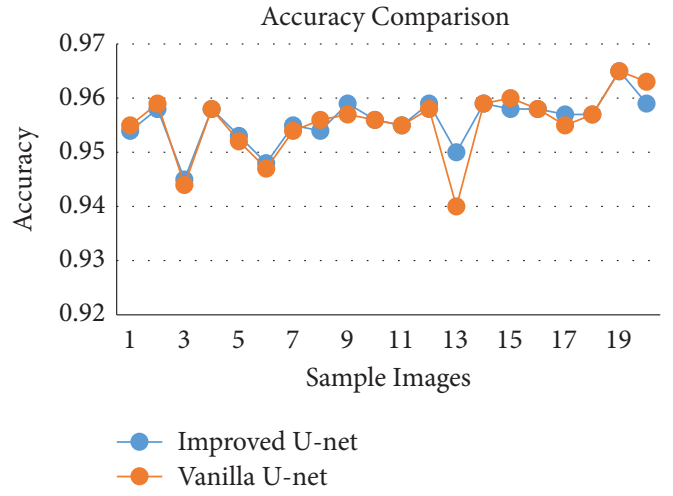

(b)

Figure 6: (a) Sensitivity comparison of U-Net with improved U-Net. (b) Accuracy comparison of U-Net with improved U-Net.

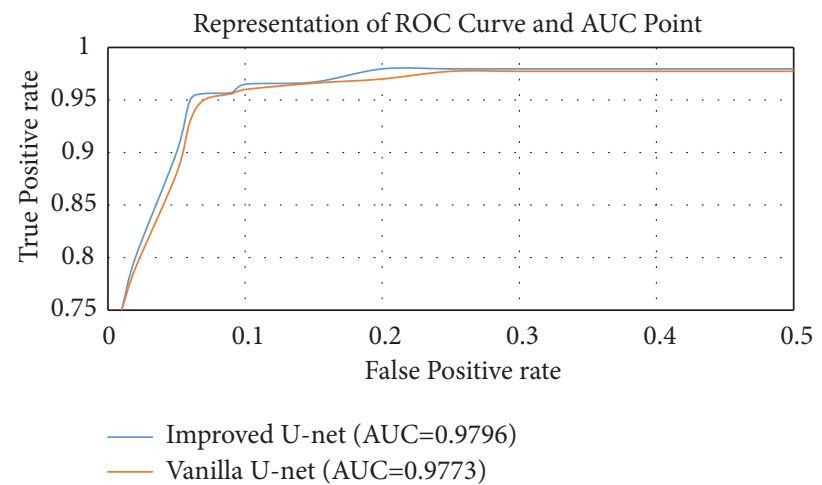

FIgURE 7: Representation of the ROC curve and AUC point at segmentation of blood vessels using the DRIVE dataset. 


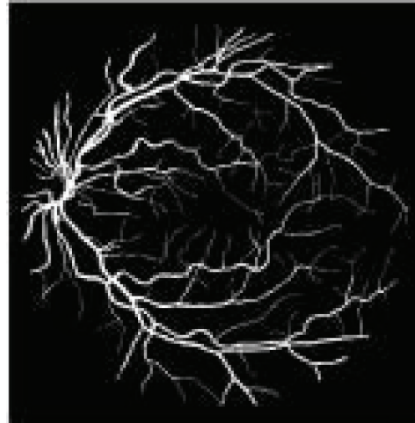

(a)

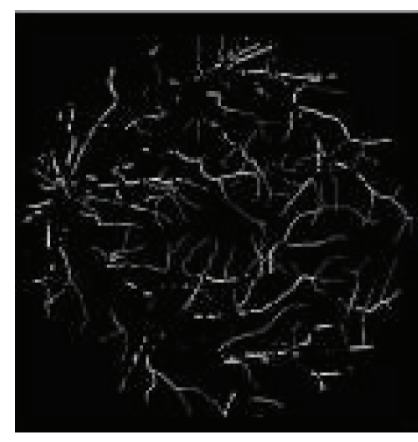

(b)

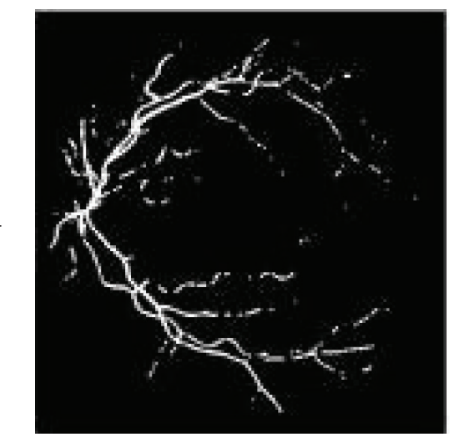

(c)

FIgURE 8: Thin and large vessel partition.

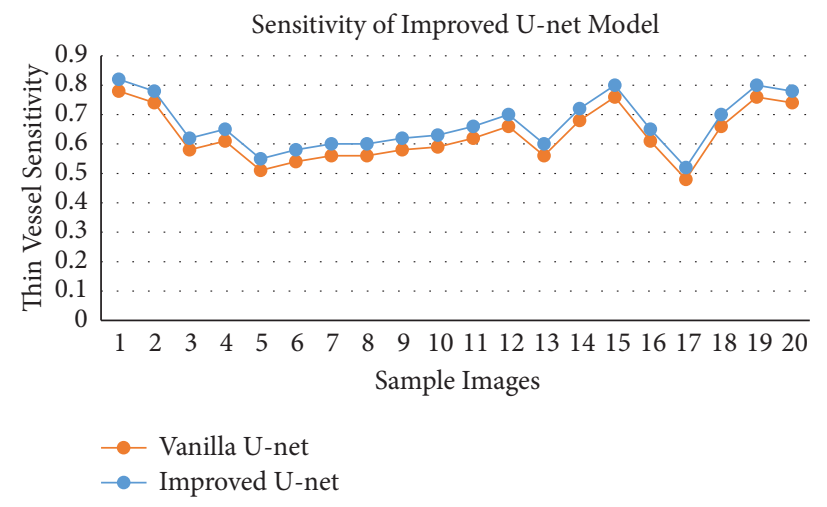

FIGURE 9: Sensitivity of the improved U-Net model.
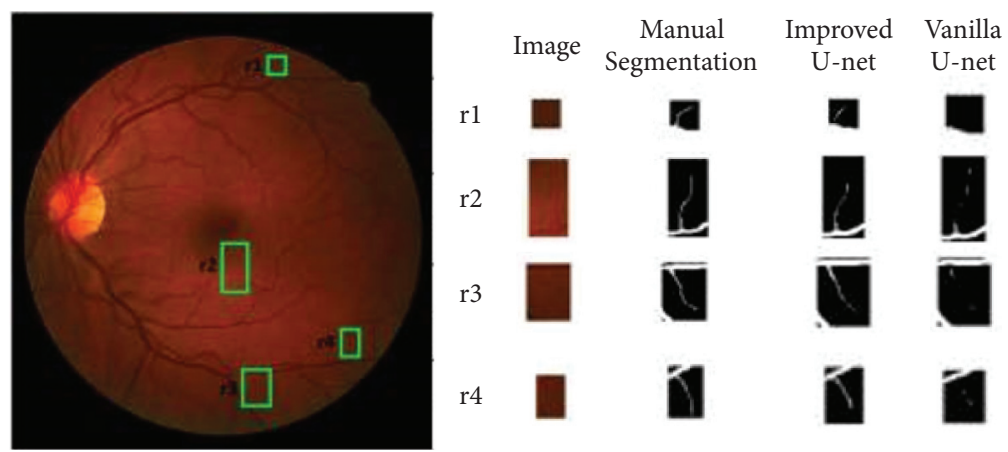

FIgURE 10: Thin vessel segmentation result representation.

segment. The computational time taken for the task by different methods is compared. We cannot compare the processing time directly and honestly because the implementation software and platform are different. However, it is shown in Figures 8 and 9 that the proposed strategy not just beats all of the existing techniques as far as sensitivity, specificity, accuracy, and geometric mean are concerned but also additionally distinguishes slender vessels successfully. For assisted diagnosis in the proposed method, segmentation achieves a better computing time of 21.562 seconds. 


\section{Conclusion}

The proposed method uses vanilla U-Net that includes multiscale inputs and dense block for segmenting retinal blood vessels from the fundus image.. Compared to the vanilla U-Net and other approaches, experimental findings show that the improved U-Net method delivers high-quality results in segmentation and computation. Furthermore, the findings of the proposed method, in particular for the segmentation of narrow arteries, indicate significant improvements. As a result, this approach is fundamental since it can help doctors diagnose fundus-related disorders. Currently, the proposed method only harvests picture patches surrounding vessel pixels as the network's input and achieves an accuracy of $95.6 \%$ in $21.56 \mathrm{sec}$. This accuracy level is comparatively higher than the traditional U-Net architecture. As a result, the modified dense conventional U-Net architecture contributes to better results in retinal blood vessel segmentation.

In the future, other data augmentation strategies will be used to improve the efficiency of medical diagnosis. They can be used not only in retinal image classifications but also can be used for other X-ray and CT images for biomedical applications to improvise the modern healthcare system for quick diagnosis $[73,74]$.

\section{Data Availability}

We can send the datasets at the request of the authors.

\section{Conflicts of Interest}

The authors declare that there are no conflicts of interest.

\section{References}

[1] G. Azzopardi, N. Strisciuglio, M. Vento, and N. Petkov, "Trainable COSFIRE filters for vessel delineation with application to retinal images," Medical Image Analysis, vol. 19, no. 1, pp. 46-57, 2015, [PubMed] [CrossRef] [Google Scholar].

[2] A. M. R. R. Bandara and P. W. G. R. M. P. B. Giragama, “A retinal image enhancement technique for blood vessel segmentation algorithm," in Proceedings of the 2017 IEEE international conference on industrial and information systems (ICIIS), pp. 1-5, IEEE, Peradeniya, Sri Lanka, 2017 December.

[3] D. A. Dharmawan, D. Li, B. P. Ng, and S. Rahardja, "A new hybrid algorithm for retinal vessels segmentation on fundus images," IEEE Access, vol. 7, pp. 41885-41896, 2019.

[4] S. Roychowdhury, D. D. Koozekanani, and K. K. Parhi, "Iterative vessel segmentation of fundus images," IEEE Transactions on Biomedical Engineering, vol. 62, no. 7, pp. 1738-1749, 2015, [PubMed] [CrossRef] [Google Scholar].

[5] E. Y. Ng, U. R. Acharya, J. S. Suri, and A. Campilho, Eds., Image Analysis and Modeling in Ophthalmology, CRC Press, Boca Raton, FL, USA, [Google Scholar], 2014.

[6] U. T. V. Nguyen, A. Bhuiyan, L. A. F. Park, and K. Ramamohanarao, "An effective retinal blood vessel segmentation method using multi-scale line detection," Pattern Recognition, vol. 46, no. 3, pp. 703-715, 2013, [CrossRef] [Google Scholar].
[7] S. Kannan, T. Kalaikumar, S. Karthik, and V. P. Arunachala, "Ant colony optimization for routing in mobile ad-hoc networks," International Journal of Soft Computing, vol. 5, no. 6, pp. 223-228, 2010.

[8] B. Sheng, P. Li, S. Mo et al., "Retinal vessel segmentation using minimum spanning superpixel tree detector," IEEE Transactions on Cybernetics, vol. 49, no. 7, pp. 2707-2719, 2018.

[9] T. A. Soomro, M. A. Khan, J. Gao, T. M. Khan, M. Paul, and N. Mir, "Automatic retinal vessel extraction algorithm," in Proceedings of the 2016 International Conference on Digital Image Computing: Techniques and Applications (DICTA), pp. 1-8, IEEE, Gold Coast, Australia, 2016 November, [CrossRef] [Google Scholar].

[10] A. Oliveira, S. Pereira, and C. A. Silva, "Retinal vessel segmentation based on fully convolutional neural networks," Expert Systems with Applications, vol. 112, pp. 229-242, 2018, [CrossRef] [Google Scholar].

[11] K. Yue, B. Zou, Z. Chen, and Q. Liu, "Improved multi-scale line detection method for retinal blood vessel segmentation," IET Image Processing, vol. 12, no. 8, pp. 1450-1457, 2018.

[12] B. Zhang, L. Zhang, L. Zhang, and F. Karray, "Retinal vessel extraction by matched filter with first-order derivative of Gaussian," Computers in Biology and Medicine, vol. 40, no. 4, pp. $438-445,2010$.

[13] J. Zhang, Y. Chen, E. Bekkers, M. Wang, B. Dashtbozorg, and B. M. t. H. Romeny, "Retinal vessel delineation using a braininspired wavelet transform and random forest," Pattern Recognition, vol. 69, pp. 107-123, 2017, [CrossRef] [Google Scholar].

[14] J. Zhang, B. Dashtbozorg, E. Bekkers, J. P. W. Pluim, R. Duits, and B. M. ter Haar Romeny, "Robust retinal vessel segmentation via locally adaptive derivative frames in orientation scores," IEEE Transactions on Medical Imaging, vol. 35, no. 12, pp. 2631-2644, 2016, [PubMed] [CrossRef] [Google Scholar].

[15] Y. Qian Zhao, X. Hong Wang, X. Fang Wang, and F. Y. Shih, "Retinal vessels segmentation based on level set and region growing," Pattern Recognition, vol. 47, no. 7, pp. 2437-2446, 2014, [CrossRef] [Google Scholar].

[16] C. Zhu, B. Zou, X. Yao, Q. Yan, Y. Liang, and J. Cui, "A survey of retinal vessel segmentation in fundus images," Journal of Computer Aided Design and Computer Graphics, vol. 27, no. 11, pp. 2046-2057, 2015, [Google Scholar].

[17] G. P. Joshi, F. Alenezi, G. Thirumoorthy, A. K. Dutta, and J. You, "Ensemble of deep learning-based multimodal remote sensing image classification model on unmanned aerial vehicle networks," Mathematics, vol. 9, no. 22, p. 2984, 2021.

[18] F. Alenezi and K. C. Santosh, "Geometric regularized hopfield neural network for medical image enhancement," International Journal of Biomedical Imaging, vol. 2021, Article ID 6664569, 2021.

[19] F. Alenezi and E. Salari, "A fuzzy-based medical image fusion using a combination of maximum selection and gabor filters," International Journal of Engineering Science, vol. 9, pp. 118129, 2018.

[20] F. S. Alenezi and S. Ganesan, "Geometric-pixel guided singlepass convolution neural network with graph cut for image d," IEEE Access, vol. 9, pp. 29380-29391, 2021.

[21] K. Hu, Z. Zhang, X. Niu et al., "Retinal vessel segmentation of color fundus images using multiscale convolutional neural network with an improved cross-entropy loss function," Neurocomputing, vol. 309, pp. 179-191, 2018, [CrossRef] [Google Scholar].

[22] Y. Zhao, L. Rada, K. Chen, S. P. Harding, and Y. Zheng, "Automated vessel segmentation using infinite perimeter 
active contour model with hybrid region information with application to retinal images," IEEE Transactions on Medical Imaging, vol. 34, no. 9, pp. 1797-1807, 2015, [PubMed] [CrossRef] [Google Scholar].

[23] T. A. Soomro, T. M. Khan, M. A. U. Khan, J. Gao, M. Paul, and L. Zheng, "Impact of ICA-based image enhancement technique on retinal blood vessels segmentation," IEEE Access, vol. 6, pp. 3524-3538, 2018.

[24] Y. Jiang, N. Tan, T. Peng, and H. Zhang, "Retinal vessels segmentation based on dilated multi-scale convolutional neural network," IEEE Access, vol. 7, pp. 76342-76352, 2019.

[25] Q. Jin, Z. Meng, T. D. Pham, Q. Chen, L. Wei, and R. Su, "DUNet: a deformable network for retinal vessel segmentation," Knowledge-Based Systems, vol. 178, pp. 149-162, 2019.

[26] C. Guo, M. Szemenyei, Y. Pei, Y. Yi, and W. Zhou, "SD-UNet: a structured dropout U-Net for retinal vessel segmentation," in Proceedings of the 2019 IEEE 19th International Conference on Bioinformatics and Bioengineering (BIBE), pp. 439-444, IEEE, Athens, Greece, 2019 October.

[27] K. B. Khan, A. A. Khaliq, and M. Shahid, "B-COSFIRE filter and VLM based retinal blood vessels segmentation and denoising," in Proceedings of the 2016 International Conference on Computing, Electronic and Electrical Engineering (ICE Cube), pp. 132-137, IEEE, Quetta, Pakistan, 2016 April.

[28] K. B. Khan, A. A. Khaliq, M. Shahid, and S. Khan, "An efficient technique for retinal vessel segmentation and denoising using modified ISODATA and CLAHE," IIUM Engineering Journal, vol. 17, no. 2, pp. 31-46, 2016.

[29] M. A. U. Khan, T. M. Khan, T. A. Soomro, N. Mir, and J. Gao, "Boosting sensitivity of a retinal vessel segmentation algorithm," Pattern Analysis \& Applications, vol. 22, no. 2, pp. 583-599, 2019.

[30] M. Z. C. Azemin, F. Abd Hamid, M. I. B. M. Tamrin, and A. H. M. Amin, "Supervised retinal vessel segmentation based on neural network using broader aging dataset," in Proceedings of the 2nd International Work-Conference on Bioinformatics and Biomedical Engineering IWBBIO, pp. 1235-1242, Granada, Spain, April 2014.

[31] B. Khomri, A. Christodoulidis, L. Djerou, M. C. Babahenini, and F. Cheriet, "Retinal blood vessel segmentation using the elite-guided multi-objective artificial bee colony algorithm," IET Image Processing, vol. 12, no. 12, pp. 2163-2171, 2018.

[32] L. Li, M. Verma, Y. Nakashima, H. Nagahara, and R. Kawasaki, "Iternet: retinal image segmentation utilizing structural redundancy in vessel networks," in Proceedings of the IEEE/CVF Winter Conference on Applications of Computer Vision, pp. 3656-3665, Waikoloa, HI, USA, January 2020.

[33] M. Li, Q. Yin, and M. Lu, "Retinal blood vessel segmentation based on multi-scale deep learning," in Proceedings of the 2018 Federated Conference on Computer Science and Information Systems (FedCSIS), pp. 1-7, IEEE, Poznan, Poland, 2018, September.

[34] Q. Li, B. Feng, L. Xie, P. Liang, H. Zhang, and T. Wang, "A cross-modality learning approach for vessel segmentation in retinal images," IEEE Transactions on Medical Imaging, vol. 35, no. 1, pp. 109-118, 2015.

[35] L. M. Liang, C. L. Huang, F. Shi, J. Wu, H. J. Jiang, and X. J. Chen, "Retinal vessel segmentation using level set combined with shape priori," Chinese Journal of Computers, vol. 41, no. 7, pp. 1678-1692, 2018.

[36] Z. Luo, Y. Zhang, L. Zhou, B. Zhang, J. Luo, and H. Wu, "Micro-vessel image segmentation based on the AD-UNet model," IEEE Access, vol. 7, pp. 143402-143411, 2019.
[37] Y. Lv, H. Ma, J. Li, and S. Liu, "Attention guided u-net with atrous convolution for accurate retinal vessels segmentation," IEEE Access, vol. 8, pp. 32826-32839, 2020.

[38] D. Maji, A. Santara, P. Mitra, and D. Sheet, "Ensemble of deep convolutional neural networks for learning to detect retinal vessels in fundus images," 2016, https://arxiv.org/abs/1603. 04833.

[39] D. Marín, A. Aquino, M. E. Gegúndez-Arias, and J. M. Bravo, "A new supervised method for blood vessel segmentation in retinal images by using gray-level and moment invariantsbased features," IEEE Transactions on Medical Imaging, vol. 30, no. 1, pp. 146-158, 2010.

[40] A. M. Mendonca and A. Campilho, "Segmentation of retinal blood vessels by combining the detection of centerlines and morphological reconstruction," IEEE Transactions on Medical Imaging, vol. 25, no. 9, pp. 1200-1213, 2006.

[41] M. Z. Alom, C. Yakopcic, M. Hasan, T. M. Taha, and V. K. Asari, "Recurrent residual U-Net for medical image segmentation," Journal of Medical Imaging, vol. 6, no. 1, Article ID 014006, 2019.

[42] L. Jin, "3AU-Net: triple attention U-net for retinal vessel segmentation," in Proceedings of the 2020 IEEE 2nd International Conference on Civil Aviation Safety and Information Technology (ICCASIT, pp. 612-615, IEEE, 2020, October.

[43] G. B. Kande, T. S. Savithri, and P. V. Subbaiah, "Retinal vessel segmentation using histogram matching," in Proceedings of the APCCAS 2008-2008 IEEE Asia Pacific Conference on Circuits and Systems, pp. 129-132, IEEE, Macao, China, 2008, November.

[44] S. Suthir and S. Janakiraman, "SNT algorithm and DCS protocols coalesced a contemporary hasty file sharing with network coding influence," Journal of Engineering Research, vol. 6, no. 3, pp. 54-69, 2018.

[45] Z. Yan, X. Yang, and K. T. Cheng, "A three-stage deep learning model for accurate retinal vessel segmentation," IEEE journal of Biomedical and Health Informatics, vol. 23, no. 4, pp. 1427-1436, 2018.

[46] X. You, Q. Peng, Y. Yuan, Y. M. Cheung, and J. Lei, "Segmentation of retinal blood vessels using the radial projection and semi-supervised approach," Pattern Recognition, vol. 44, no. 10-11, pp. 2314-2324, 2011.

[47] O. Oktay, J. Schlemper, L. L. Folgoc et al., "Attention u-net: learning where to look for the pancreas," 2018, https://arxiv. org/abs/1804.03999.

[48] M. U. Akram, A. Atzaz, S. F. Aneeque, and S. A. Khan, "Blood vessel enhancement and segmentation using wavelet transform," in Proceedings of the 2009 International Conference on Digital Image Processing, pp. 34-38, IEEE, Bangkok, Thailand, 2009, March.

[49] S. Jégou, M. Drozdzal, D. Vazquez, A. Romero, and Y. Bengio, "The one hundred layers tiramisu: fully convolutional densenets for semantic segmentation," in Proceedings of the IEEE conference on computer vision and pattern recognition workshops, pp. 11-19, Honolulu, HI, USA, July 2017.

[50] W. S. Oliveira, J. V. Teixeira, T. I. Ren, G. D. C. Cavalcanti, and J. Sijbers, "Unsupervised retinal vessel segmentation using combined filters," PloS one, vol. 11, no. 2, p. e0149943, 2016.

[51] J. I. Orlando and M. Blaschko, "Learning fully-connected CRFs for blood vessel segmentation in retinal images," in Proceedings of the International Conference On Medical Image Computing And Computer-Assisted Intervention, pp. 634-641, Springer, Virtual Event, September 2014.

[52] Q. Peng, X. You, L. Zhou, and Y. M. Cheung, "Retinal blood vessels segmentation using the radial projection and 
supervised classification," in Proceedings of the 2010 20th International Conference on Pattern Recognition, pp. 14891492, IEEE, Istanbul, Turkey, 2010, August.

[53] K. Shanmugam, K. Subburathinam, and A. Velayuthampalayam Palanisamy, "A dynamic probabilistic based broadcasting scheme for manets," The Scientific World Journal, vol. 2016, Article ID 1832026, 8 pages, 2016.

[54] O. Ali, N. Muhammad, Z. Jadoon, B. M. Kazmi, N. Muzamil, and Z. Mahmood, "A comparative study of automatic vessel segmentation algorithms," in Proceedings of the 2020 3rd International Conference on Computing, Mathematics and Engineering Technologies (iCoMET), pp. 1-6, IEEE, Sukkur, Pakistan, 2020 January.

[55] G. S. Ramlugun, V. K. Nagarajan, and C. Chakraborty, "Small retinal vessels extraction towards proliferative diabetic retinopathy screening," Expert Systems with Applications, vol. 39, no. 1, pp. 1141-1146, 2012, [CrossRef] [Google Scholar].

[56] O. Ronneberger, P. Fischer, and T. Brox, "U-net: convolutional networks for biomedical image segmentation," in Proceedings of the International Conference on Medical image computing and computer-assisted intervention, pp. 234-241, Springer, Munich, Germany, October 2015.

[57] S. Roychowdhury, D. D. Koozekanani, and K. K. Parhi, "Blood vessel segmentation of fundus images by major vessel extraction and subimage classification," IEEE journal of biomedical and health informatics, vol. 19, no. 3, pp. 11181128, 2014, [PubMed] [CrossRef] [Google Scholar].

[58] D. Koukounis, C. Ttofis, A. Papadopoulos, and T. Theocharides, "A high performance hardware architecture for portable, low-power retinal vessel segmentation," Integration, vol. 47, no. 3, pp. 377-386, 2014, [CrossRef] [Google Scholar].

[59] T. A. Soomro, M. A. U. Khan, J. Gao, T. M. Khan, and M. Paul, "Contrast normalization steps for increased sensitivity of a retinal image segmentation method," Signal, Image and Video Processing, vol. 11, no. 8, pp. 1509-1517, 2017, [CrossRef] [Google Scholar].

[60] J. Staal, M. D. Abramoff, M. Niemeijer, M. A. Viergever, and B. Van Ginneken, "Ridge-based vessel segmentation in color images of the retina," IEEE Transactions on Medical Imaging, vol. 23, no. 4, pp. 501-509, 2004.

[61] N. Strisciuglio, G. Azzopardi, M. Vento, and N. Petkov, "Supervised vessel delineation in retinal fundus images with the automatic selection of B-COSFIRE filters," Machine Vision and Applications, vol. 27, no. 8, pp. 1137-1149, 2016, [CrossRef] [Google Scholar].

[62] T. Laibacher, T. Weyde, and S. Jalali, "M2u-net: effective and efficient retinal vessel segmentation for real-world applications," in Proceedings of the IEEE/CVF Conference on Computer Vision and Pattern Recognition Workshops, p. 0, Long Beach, CA, USA, June 2019.

[63] Q. Wang, Z. Yuan, Q. Du, and X. Li, "GETNET: a general endto-end 2-D CNN framework for hyperspectral image change detection," IEEE Transactions on Geoscience and Remote Sensing, vol. 57, no. 1, pp. 3-13, 2018.

[64] S. Wang, Y. Yin, G. Cao, B. Wei, Y. Zheng, and G. Yang, "Hierarchical retinal blood vessel segmentation based on feature and ensemble learning," Neurocomputing, vol. 149, pp. 708-717, 2015.

[65] X. Wang, X. Jiang, and J. Ren, "Blood vessel segmentation from fundus image by a cascade classification framework," Pattern Recognition, vol. 88, pp. 331-341, 2019.

[66] X. H. Wang, Y. Q. Zhao, M. Liao, and B. Zou, "Automatic segmentation for retinal vessel based on multi-scale 2D Gabor wavelet," Acta Automatica Sinica, vol. 41, no. 5, pp. 970-980, 2015.

[67] W. Xiancheng, L. Wei, M. Bingyi et al., "Retina blood vessel segmentation using a U-net based Convolutional neural network," in Proceedings of the Procedia Computer Science: International Conference on Data Science (ICDS 2018), pp. 8-9, Beijing, China, June 2018.

[68] Z. Yan, X. Yang, and K.-T. Cheng, "Joint segment-level and pixel-wise losses for deep learning based retinal vessel segmentation," IEEE Transactions on Biomedical Engineering, vol. 65, no. 9, pp. 1912-1923, 2018, [PubMed] [CrossRef] [Google Scholar].

[69] P. Elumalaivasan, S. Suthir, S. Ravikumar, V. Pandiyaraju, and T. Munirathinam, "CBIR: retrieval of similar images using median vector algorithm," in Proceedings of the 2013 International Conference on Green Computing, Communication and Conservation of Energy (ICGCE), pp. 1-5, IEEE, Chennai, India, 2013, December, [CrossRef] [Google Scholar].

[70] X. Yin, B. W.-H. Ng, J. He, Y. Zhang, and D. Abbott, "Accurate image analysis of the retina using hessian matrix and binarisation of thresholded entropy with application of texture mapping," PLoS one, vol. 9, no. 4, [PMC free article] [PubMed] [CrossRef] [Google Scholar], Article ID e95943, 2014.

[71] Y. Guo, Ü. Budak, L. J. Vespa, E. Khorasani, and A. Şengür, “A retinal vessel detection approach using convolution neural network with reinforcement sample learning strategy," Measurement, vol. 125, pp. 586-591, 2018, [CrossRef] [Google Scholar].

[72] M. M. Fraz, P. Remagnino, A. Hoppe et al., "An ensemble classification-based approach applied to retinal blood vessel segmentation," IEEE Transactions on Biomedical Engineering, vol. 59, no. 9, pp. 2538-2548, 2012, [PubMed] [CrossRef] [Google Scholar].

[73] J. Hu, H. Wang, S. Gao et al., "S-unet: a bridge-style u-net framework with a saliency mechanism for retinal vessel segmentation," IEEE Access, vol. 7, pp. 174167-174177, 2019.

[74] K. B. Khan, A. A. Khaliq, A. Jalil, and M. Shahid, "A robust technique based on VLM and Frangi filter for retinal vessel extraction and denoising," PLoS One, vol. 13, no. 2, Article ID e0192203, 2018. 